\title{
Cherenkov interaction and post-acceleration experiments of high brightness electron beams from a pseudospark discharge
}

\author{
H. Yin*, A.W. Cross, A.D.R. Phelps, W. He, K. Ronald \\ Department of Physics, University of Strathclyde, Glasgow G4 ONG, UK
}

\begin{abstract}
A pseudospark-sourced electron beam has two phases, an initial hollow cathode phase (HCP) beam followed by a conductive phase (CP) beam. The beam brightness was measured by a field-free collimator to be $10^{9}$ and $10^{11} \mathrm{~A} \mathrm{~m}^{-2} \mathrm{rad}^{-2}$ for HCP beam and CP beam, respectively. The initial HCP beam from an eight-gap pseudospark discharge was applied in a Cherenkov interaction between the electron beam and the $\mathrm{TM}_{01}$ mode of a $60-\mathrm{cm}$ long alumina-lined waveguide. While the CP beam from a three-gap pseudospark discharge chamber was propagated and post-accelerated from about $200 \mathrm{~V}$ to more than $40 \mathrm{kV}$.
\end{abstract}

Keywords: Pseudospark discharge; High brightness; Electron beam; Cherenkov interaction; Post-acceleration

\section{Introduction}

Pseudospark discharge [1-3] experiments to generate a low-temperature plasma for use as a copious source of electrons have been carried out at the University of Strathclyde. The plasma can be regarded as a low work function surface that facilitates electron extraction. Initial study of electron beam production was carried out on a single-gap pseudospark system for a wide range of parameters, including cathode cavity length, cathode hole size, applied voltage, external capacitance and the inductance in the discharge circuit [4]. Higher-energy electron beam production, more suitable for high power microwave generation, was studied using multi-gap pseudospark systems. Electron beam pulses of duration of tens of ns, current density $\left(>10^{8} \mathrm{~A} \mathrm{~m}^{-2}\right.$ ), brightness of up to $10^{12} \mathrm{~A} \mathrm{~m}^{-2} \mathrm{rad}^{-2}$ and emittance of tens of $\mathrm{mm} \mathrm{mrad}$ were measured from a pseudospark discharge [5]. This beam has a higher combined current density and brightness compared to electron beams formed from any other known type of electron source. It was applied in a Cherenkov maser interaction and also transported in a plasma-induced ion background and simultaneously accelerated by an accelerating potential. This article will present some results from these experiments. 


\section{Cherenkov maser interaction experiment}

A schematic outline of the pseudospark-based Cherenkov maser amplifier is shown in Fig. 1. The main components of the experiment are the pseudospark-based electron beam source, the magnetic field for beam transport, the Cherenkov interaction region, electrical/beam diagnostics and the microwave launching/diagnostic system. In the maser system, the presence of the dielectric in the waveguide reduces the phase velocity of the electromagnetic waves, allowing a resonant interaction to occur between a TM or HE waveguide mode and the rectilinear electron beam. Coherence of the generated radiation arises due to bunching of electrons in phase with respect to the electromagnetic wave.

Microwave radiation was detected successfully from the pseudospark-based dielectric Cherenkov maser amplifier. The temporal profile of the microwave output radiation from the maser is shown in the lower part of Fig. 2, time correlated with the electron-beam current and voltage profiles.

The frequency range of the microwave radiation from the Cherenkov maser amplifier was measured to be between 25.5 and $28.6 \mathrm{GHz}$ by applying different cylindrical cut-off filters in the waveguide. The observed frequency was found to be $20 \%$ higher than that predicted by the resonance condition for $70-80 \mathrm{keV}$ beam energy. This discrepancy is probably due to charging of the dielectric liner [6].

The output antenna pattern associated with the azimuthal E-field component was measured to be independent of the presence of the dielectric and close to zero, confirming the operation of a TM mode. The measured pattern was in good agree-

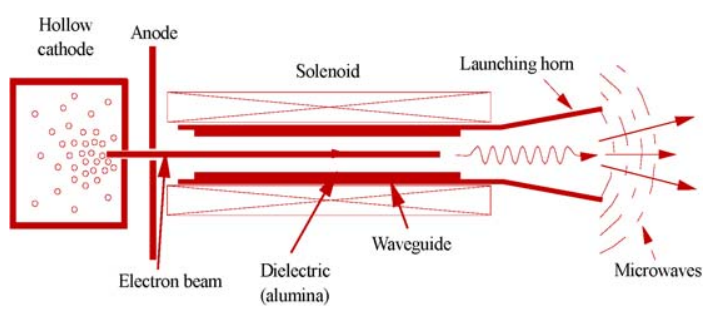

Fig. 1. Cherenkov maser experimental configuration.

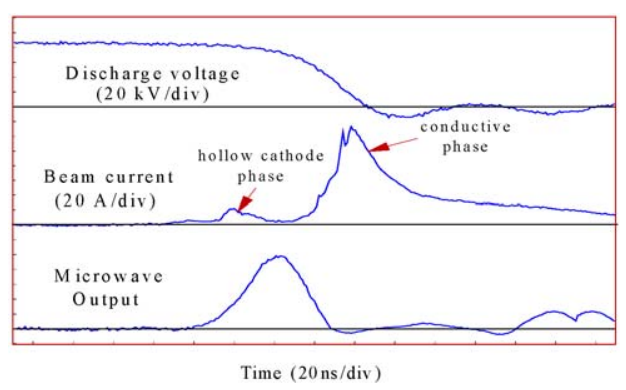

Fig. 2. Typical waveforms of pseudospark discharge voltage, the beam current and the microwave pulse.

ment with the results from bench experiments in which a $27 \mathrm{GHz} \quad \mathrm{TM}_{01}$ microwave signal was launched using the same horn. This confirms the operation mode to be $\mathrm{TM}_{01}$ mode. The peak power was measured to be around $2 \pm 0.2 \mathrm{~kW}$ and the gain to be $29 \pm 3 \mathrm{~dB}$. A relative spectral energy distribution was obtained and approximately $65 \%$ of the radiation was found to lie in $25-28.6 \mathrm{GHz}$ frequency band.

To complement the experimental investigations of the Cherenkov maser, a three-dimensional numerical simulation code was developed [7,8]. The simulations show that the $\mathrm{TM}_{01}$ mode at $\sim 21 \mathrm{GHz}$ is amplified strongly, attaining a power of $\sim 3.4 \mathrm{~kW}$ at $z=60 \mathrm{~cm}$, whereas the power in the $\mathrm{TM}_{02}$ mode at $\sim 55 \mathrm{GHz}$ remains around its initial level. These simulations support the interpretation of the experimental results as microwave amplification via a Cherenkov interaction between the high-quality electron beam and the $\mathrm{TM}_{01}$ mode of the dielectric-lined waveguide.

\section{Post-acceleration experiment}

A schematic outline of the experimental setup for the study of the propagation and the postacceleration of the pseudospark-sourced beam is shown in Fig. 3. The electron beam was extracted from a three-gap pseudospark discharge chamber. The single Perspex disc of inner diameter $5 \mathrm{~mm}$, outer diameter $300 \mathrm{~mm}$ and 26-mm thickness was used for acceleration gap insulation. It was made with a recess to achieve a 5-mm acceleration gap separation. 


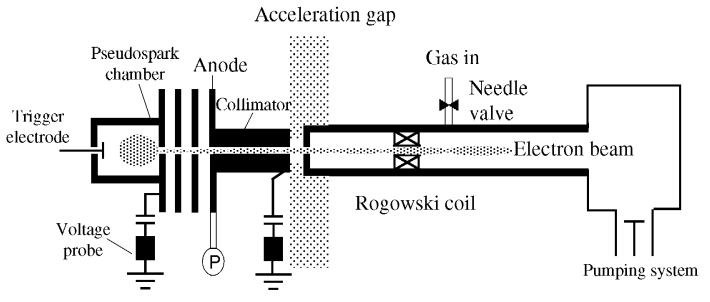

Fig. 3. Schematic diagram for the post-acceleration experiment of the pseudospark electron beam.

The pseudospark discharge was powered by a DC power supply. The hollow cathode was connected through a $30 \mathrm{M} \Omega$ charging resistor to a negative voltage source $(-30 \mathrm{kV}, 1 \mathrm{~mA}$ DC power supply) and the charging voltage was measured by a capacitive voltage probe. The external energy storage capacitance was $600 \mathrm{pF}$. The acceleration unit was driven by a $40 \mathrm{kV}, 125 \mathrm{~ns}$ voltage pulse produced by a cable Blumlein and the acceleration voltage was measured by another capacitive voltage probe. The pseudospark discharge and the cable Blumlein were triggered by two sets of trigger signals controlled by two delay units and one trigger source. Both trigger signals for the pseudospark and the cable Blumlein were $15 \mathrm{kV}$ pulses. Careful adjustment of the delay units ensured the beam acceleration voltage was applied at the right time.

The propagation of the electron beam from a three-gap pseudospark discharge chamber was studied as a function of the length of a collimator of $3.5-\mathrm{mm}$ internal diameter. Rogowski coil beam current measurements $150 \mathrm{~mm}$ downstream from the anode showed that with no magnetic guiding field, $70 \%$ and $50 \%$ of the beam propagated through 30 and $60 \mathrm{~mm}$ long collimators, respectively. A magnetic field free collimator technique also enabled a beam brightness of up to $10^{11-12} \mathrm{~A} \mathrm{~m}^{-2} \mathrm{rad}^{-2}$ to be measured from a 3-gap pseudospark discharge.

In the post-acceleration experiment a $30 \mathrm{~mm}$ long collimator of $3.5 \mathrm{~mm}$ internal diameter was used after the anode of the pseudospark discharge chamber and the acceleration unit was located immediately after the collimator to achieve a gas pressure gradient and to optimize beam current. The beam acceleration experiments showed that careful adjustment of the trigger system could ensure synchronization between the beam propagation and the application of the acceleration voltage. A $100 \mathrm{~A}, 40 \mathrm{kV}$ electron beam pulse was measured at a distance of $120 \mathrm{~mm}$ from the acceleration gap without a magnetic guiding field, as shown in Fig. 4. However, in Fig. 4 some beam loading effect is evident where it can be seen that as the beam current increases the post acceleration voltage decreases and the flat top of the voltage signal becomes shorter. During the experiments, the beam loading effect was mitigated by reducing the internal impedance of the cable pulser from 50 to $14 \Omega$. It is possible to further reduce the beam loading effect by continuing to decrease the internal impedance of the cable Blumlein or by using an alternative lower impedance pulse forming line.

Beam propagation across the acceleration gap and further along the beam channel was simulated using the electromagnetic PIC code MAGIC. The simulations show that a $200 \mathrm{~V}, 200 \mathrm{~A}$ beam in a pseudospark CP phase will propagate across a $40 \mathrm{kV}$ post-acceleration gap of $5 \mathrm{~mm}$ separation in an ion background of certain densities. About $10 \%$ and $70 \%$ of the beam would propagate across the gap when the ion densities are $1 \times 10^{12}$ and $6 \times 10^{12} \mathrm{~cm}^{-3}$, respectively. Fig. 5 shows a simulated beam profile and current variation during its propagation across the acceleration gap in an ion background of density $6 \times 10^{12} \mathrm{~cm}^{-3}$.

The simulation also shows that with ion background, the shapes of both the cathode and anode of the acceleration gap have little effect on beam propagation.

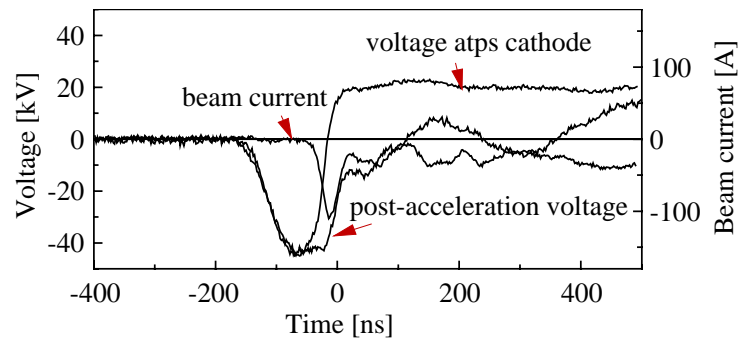

Fig. 4. Typical record of the time-correlated pseudospark discharge voltage, beam current and the acceleration voltage pulse. 

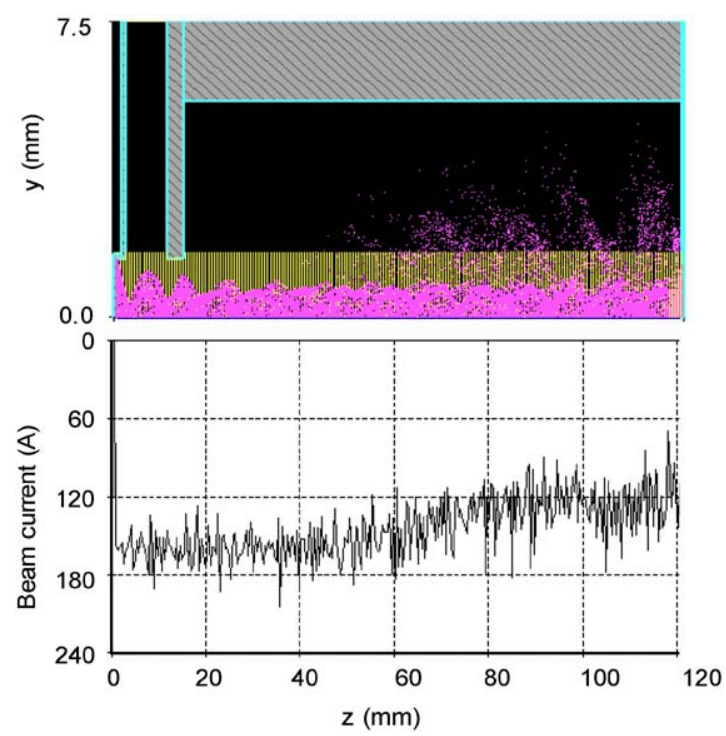

Fig. 5. Simulated beam profile and current variation during propagation across the acceleration gap and further along the beam channel with an ion background of density $6 \times 10^{12} \mathrm{~cm}^{-3}$ (K, cathode; A, anode; $z$, beam channel axis; vertical dense lines, plasma zone; and cloud, electrons).

\section{Discussion and conclusions}

In conclusion, we have presented measurements of coherent electromagnetic radiation generation in a free electron maser using an electron beam from a pseudospark discharge. The microwave radiation was generated by Cherenkov amplification of the broadband emission from the pseudospark discharge. The frequency of the microwave output after the Cherenkov maser interaction was measured to be mainly around $25.5 \mathrm{GHz}$ and the dominating mode was identified as being $\mathrm{TM}_{01}$. The peak power is of around $2 \pm 0.2 \mathrm{~kW}$ and the gain was measured as $29 \pm 3 \mathrm{~dB}$.
A $100 \mathrm{~A}, 40 \mathrm{kV}$ electron beam pulse was measured at a distance of $120 \mathrm{~mm}$ from the acceleration gap without a magnetic guiding field. Comparing this with the simulation implies that a favorable ion background exists along the beam channel and the acceleration gap. The ion background can be formed from the background gas ionization by the initial high energy HCP beam during the pseudospark discharge, which also expels electrons in the ionized gas media. In summary, the beam in the pseudospark conductive phase was successfully accelerated from about $200 \mathrm{~V}$ to more than $40 \mathrm{kV}$.

\section{Acknowledgements}

The authors would also like to thank the Engineering and Physical Sciences Research Council (EPSRC) for supporting this work.

\section{References}

[1] J. Christiansen, C. Schultheiss, Z. . Phys. A290 (1979) 35.

[2] M.A. Gunderson, G. Schaefer, NATO ASI Ser. B, Plenum, New York, 1990.

[3] K. Frank, J. Christiansen, IEEE Trans. Plasma Sci. 17 (1989) 748.

[4] H. Yin, W. He, A.W. Cross, A.D.R. Phelps, K. Ronald, J. Appl. Phys. 90 (2001) 3212.

[5] H. Yin, G.R.M. Robb, W. He, A.D.R. Phelps, A.W. Cross, K. Ronald, Phys. Plasmas 7 (2000) 5195.

[6] E. Garate, R. Cook, P. Heim, R. Layman, J.E. Walsh, J. Appl. Phys. 58 (1985) 627.

[7] H. Yin, A.D.R. Phelps, W. He, G.R.M. Robb, K. Ronald, P. Aitken, B.W.J. McNeil, A.W. Cross, C.G. Whyte, Nucl. Instr. And Meth. A 407 (1998) 175.

[8] H.P. Freund, Phys. Rev. Lett. 65 (1990) 2989. 